\title{
Automated handling and assembly of customizable AFM-tips
}

Bartenwerfer, M.; Eichhorn, V.; Jasper, D.; Fatikow, S.; Savenko, Alexey; Petersen, Dirch Hjorth; Malm, Bjarke; Bøggild, Peter

Published in:

2011 IEEE International Symposium on Assembly and Manufacturing

Link to article, DOI:

10.1109/ISAM.2011.5942350

Publication date:

2011

Link back to DTU Orbit

Citation (APA):

Bartenwerfer, M., Eichhorn, V., Jasper, D., Fatikow, S., Savenko, A., Petersen, D. H., Malm, B., \& Bøggild, P. (2011). Automated handling and assembly of customizable AFM-tips. In 2011 IEEE International Symposium on Assembly and Manufacturing IEEE. https://doi.org/10.1109/ISAM.2011.5942350

\section{General rights}

Copyright and moral rights for the publications made accessible in the public portal are retained by the authors and/or other copyright owners and it is a condition of accessing publications that users recognise and abide by the legal requirements associated with these rights.

- Users may download and print one copy of any publication from the public portal for the purpose of private study or research.

- You may not further distribute the material or use it for any profit-making activity or commercial gain

- You may freely distribute the URL identifying the publication in the public portal

If you believe that this document breaches copyright please contact us providing details, and we will remove access to the work immediately and investigate your claim. 


\section{Automated Handling and Assembly of Customizable AFM-Tips}

\author{
M. Bartenwerfer, V. Eichhorn, D. Jasper, and S. Fatikow \\ Division of Microrobotics and Control Engineering \\ University of Oldenburg \\ Oldenburg, Germany \\ m.bartenwerfer@uni-oldenburg.de
}

\author{
A. Savenko, D. H. Petersen, B. Malm, and P. Bøggild \\ Department of Micro- and Nanotechnology \\ Technical University of Denmark \\ DK-2800 Kongens Lyngby, Denmark
}

\begin{abstract}
Today's processes in micro- and nanofabrication include several critical dimension metrology steps to guarantee device performance. Especially in the manufacturing process of novel disruptive photonic devices and nanoelectronic circuit architectures, new 3D acquisition and visualization techniques for metrology are required. Two of the most important parameters are the line width and sidewall roughness of vertical interconnects and nanooptical structures. The measurement of these parameters becomes increasingly challenging as the continuous shrinking of dimensions requires higher lateral resolution. The AFM has become a standard and widely spread instrument for characterizing such nanoscale devices and can be found in most of today's research and development areas. However, the characterization of three dimensional high-aspect ratio and sidewall structures is still a bottleneck. Novel exchangeable and customizable scanning probe tips, so-called NanoBits, can be attached to standard AFM cantilevers offering unprecedented freedom in adapting the shape and size of the tips to the surface topology of the specific application. In order to realize the in-situ exchange of NanoBits within the AFM environment the NanoBits have to be provided in a freestanding way that allows the AFM cantilever to be aligned and connected to the NanoBits. Due to the fact that direct microfabrication of such structures is still challenging, a nanorobotic preassembly of NanoBits cartridges is reasonable. These cartridges are intended to contain several NanoBits with a variety of different tip-shapes.
\end{abstract}

Keywords-nano-assembling; automation; AFM-tip; customizable; characterization; optical waveguide

\section{INTRODUCTION}

Industries in micro- and nanotechnology produce devices, which are supposed to have well-defined properties in order to guarantee their performance. To achieve and keep these properties, various metrology steps for process control are required and especially measurements at the critical dimension - meaning where the system reaches its resolution limit - gain importance. In recent years, the production and development of integrated optical devices with optical waveguides and complex photonic systems have become one of the most crucial tasks in micro- and nanosystems technology.

For these components, not only surface roughness measurements on horizontal flat zones, but also measurements on vertical surfaces and on the width of groves are important. On account of the miniaturization, all these tasks will become even more challenging.
The Atomic Force Microscope (AFM) has become a standard and widely spread instrument in industry and research for the characterization of nanoscale devices. However, the capabilities of the AFM are still limited by the geometry of AFM-tips which only allows the analysis of horizontal surfaces with low aspect-ratios. A variety of research projects tackle these problems, aiming for an general improvement of AFM-cantilevers. Several works have shown, that refinement or decoration of AFM-tips can improve the imaging quality -especially all the aspect-ratio- by orders of magnitudes [1] [3].

To date, roughness measurements on vertical or even overhanging surfaces are hardly feasible. The most apparent reason is that it is impossible to bring the cantilever tip into contact with these points within the given geometrical conditions. Furthermore, controlling methods for scanning movements in planes with arbitrary orientation are still lacking.

This contribution deals with the development of novel, exchangeable, and customizable AFM-tips. These tips - called NanoBits - can be mounted at the tip of a conventional AFM-cantilever or at the end of a conventional tipless AFMcantilever. Thus, they are optionally designed to be integrated within a commercial AFM system. NanoBits offer entirely new possibilities to design the shape and size of scanning tips. Almost any orientation of the tip is possible and even multi-tip NanoBits are possible. Thus, NanoBits prove a more detailed characterization of complex structures than has been achieved to date [4].

A fundamental goal of this contribution is the development of an automated in-situ exchange of NanoBits, since this guarantees seamless integration into conventional AFM systems and processes. However, the assembly of a NanoBit with an AFM-cantilever imposes certain conditions on the setup:

- The NanoBits have to be freestanding, accessible, and perpendicular to the carrying substrate.

- The NanoBits have to be in a well-known position to enable the alignment and assembly of NanoBit and Cantilever.

The direct microfabrication of such freestanding structures is still challenging while a nanorobitic preassembly approach is very promising for the industrial exploitation. Several NanoBits can be stored in a cartridge to make them accessible 


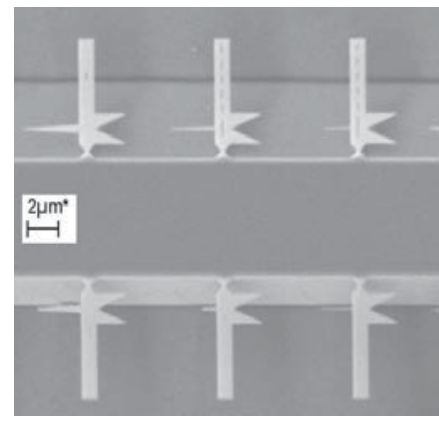

Fig. 1: SEM image of a NanoBit substrate. Each NanoBit is suspended to the silicon beam at a single point and free pending above the substrate's surface.

for the subsequent assembly. Every cartridge can contain different NanoBits for different applications and scanning tasks.

The transfer of the NanoBits from the production substrate to the cartridge is performed by special structured microgrippers. For this kind of application, handling approaches based on microgrippers allow a high throughput [5]. In combination with a cost-effective microrobotic system that might be provided together with the AFM or NanoBits, such cartridges can be integrated into the AFM environment enabling an easy and in-situ exchange of NanoBits AFM probes. The entire sequence of a NanoBit's production and application contains four main stages:

i) The lithographic fabrication of the NanoBits.

ii) The transfer from the fabrication substrate to a cartridge.

iii) The transfer from the cartridge to the tip of a cantilever.

iv) The removal of the NanoBit from the cantilever's tip

Due to deformations at all scanning probe tips, multiple usage of a single NanoBit is neither necessary nor intended.

Section II of this contribution gives a short introduction to the NanoBits and their fabrication possibilities. Section III explains the fundamental handling strategies in detail as well as experimental results and the closing Section IV gives a short overview of the used and intended automation techniques.

\section{NANOBITS: CUSTOMIZABLE AFM-TIPS}

The tip morphology of the NanoBits can be accurately shaped to match the individual application. In order to conduct AFM scanning in special modes (sidewall roughness, overhanging edges, high aspect ratio) a particular shape of the tip is typically required. In previous work, NanoBits have been prepared by electron beam lithography (EBL) and standard silicon processing. In this way, NanoBits could be produced suspended on a tiny contact and free lying above the substrate (Fig. 1). The dimensions are $2-5 \mu \mathrm{m}$ long and $120-150 \mathrm{~nm}$ thick, while the length of the handle is user-definable [6].

\section{A. Precise fabrication of NanoBits by FIB}

In this work, a second approach, focused ion beam (FIB) milling is used for rapid fabrication of custom-made prototype
AFM tips with a very short turn-around time. The enduser can then easily prepare his own tip with an applicationspecific shape in less than half an hour, without access to a cleanroom. A focused gallium ion beam at a Helios NanoLab micromachining system is used to manufacture NanoBits in a $130 \mathrm{~nm}$ thin silicon membrane (see Figure 2) [7]. The focus of this study is to obtain a precisely manufactured geometry despite well-known focused ion beam milling issues like drift and redeposition. Finally, the fabrication tolerance can approach to tens of nanometers.

The resulting shape of the structure depends significantly on the sequence in which the different parts of the pattern are milled, i.e. the milling strategy [8]. The most simple raster scanning turned out to be highly sensitive to drift and redeposition of sputtered material, which results in severe shape distortion. The drift can be caused by thermal instability of the system, residual movement of the stage, charging effects of the sample and other factors. The resolution of high end FIB milling approaches a level where residual electro-magnetic interference is difficult to compensate by regular measures such as interference interlock of power supply and antiphase suppressing coils installed in the laboratory. Usually a small drift of the milled pattern relative to the sample occurs due to several reasons. The sample stage may continue to drift immediately after a previous movement has been performed, but this effect usually quickly diminishes. Temperature drift of the equipment construction and electronics is a more persistent problem. Furthermore, placing the sample on sticky carbon tape or other mechanically instable supporters may cause creep or drift. For some types of samples electrical charging can be a problem as well. While such effects can be reduced to some extent, the small residual drift is typically unpredictable as it depends on many factors (see Figure 3).

Different patterning strategies are used in the endeavor of minimizing shape distortions [8]. The main points are the following: the milling order is automatically organized to mill the critical areas last, which reduces problems with redeposition. This is done by defining a multiple circular milling pattern with a center in each critical area of the structure, as shown in Figure 4a. Moreover, an automatic back scan of the already

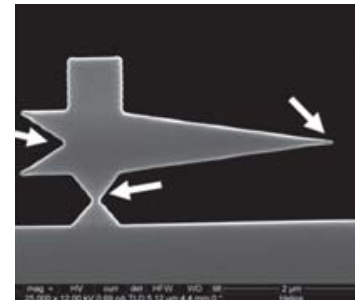
areas, i.e. the parts with the highest requirements in terms of accuracy. (a) Arrows show the critical

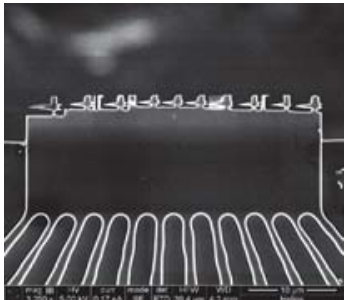
in the suspended $\mathrm{Si}$ membrane. (b) A set of NanoBits prepared

Fig. 2: SEM views of NanoBits prepared by FIB etching in the Si membrane. 


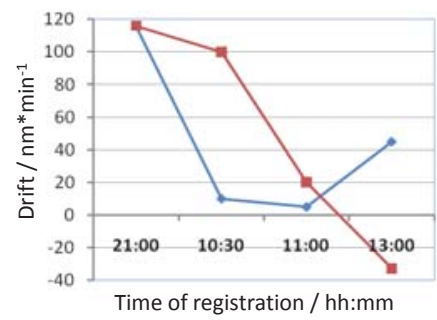

Fig. 3: Drift velocity of the pattern as a function of time.

milled contour removes redeposited material. A serial snakelike mill order of the edge is used to eliminate artifacts induced by the movement of the beam; occasionally traces are etched on the surface because of incomplete blanking (suppression) of the ion beam. The best and most robust, design-independent strategy turned out to be a combination of the above-mentioned strategy elements (see Figure 4b). This results in a significantly more uniform and geometrically exact shape of the structure. In addition to the drift of the pattern, a minor drift of the focus was observed. To counteract this, the objective lens voltage must be manually corrected by roughly $20 \mathrm{~V}$ per hour to maintain a correct focus, with the maximum value being about $19 \mathrm{kV}$. This corresponds to a $20 \mathrm{~nm}$ defocusing of the beam spot at $28 \mathrm{pA}$ beam current.

Sharpening the NanoBit tips for high resolution scanning probe microscopy using a focused ion beam is a challenging problem. Tip diameters of $30-40 \mathrm{~nm}$ are easily obtained by FIB, but $10 \mathrm{~nm}$ or less is typically required for AFMmeasurements. TEM imaging helps to evaluate the acquired shape and dimensions of the NanoBits, observe changes in the crystal structure as well as possible formation of amorphous layer from redeposition during the milling process, and also to understand underlying tip formation mechanisms [7]. Figure 5 a shows an example in which the sharpening of the tip has led to structural damage, possibly due to overheating or excessive amorphisation. The smallest tip diameter obtained by simple FIB milling from the side is approximately $23 \mathrm{~nm}$ (Fig. 5b). In the following experiment an accelerating voltage of $30 \mathrm{kV}$ was used, and an ion beam current of $28 \mathrm{pA}$. For better sharpening, it is preferable to etch along a tangent from the base towards the end of the needle shaped tip. Other sharpening strategies

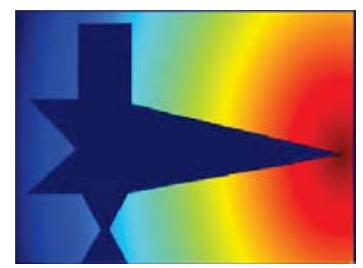

(a) Circular etch ending at the tip. The colors show the sequence of etching with light blue areas being milled first and red areas being milled last.

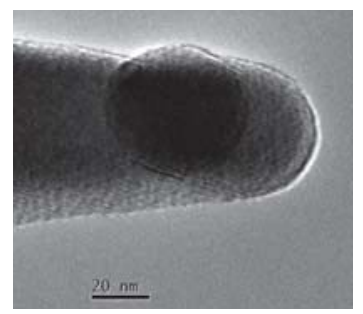

(a) TEM image shows example of Si tip formation after FIB milling (47 $\mathrm{nm}$ diameter)

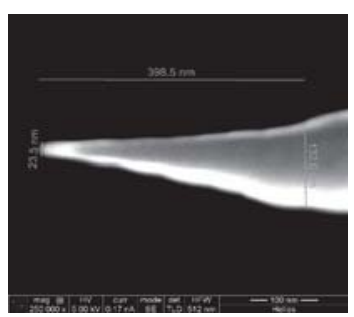

(b) Tip of the smallest diameter that was obtained: $23,5 \mathrm{~nm}$.
Fig. 5: NanoBit tips in TEM and SEM.

will also be investigated.

\section{Assembly And InTEgRation OF NANOBits AFM PROBES}

The preassembly sequence makes high demands on the setup, since it has to be assured that the NanoBits tips remain undamaged during the entire process. Thus, high positioning accuracy in nanometer range is required for the manipulation. High resolution visual feedback serves as monitoring for the handling process as well as quality control unit. In order to fulfill all these requirements, it is reasonable to mount the robotic setup inside a scanning electron microscope (SEM). It provides sufficient room for all components and a highcapacity imaging system with the accuracy of few nanometers [9].

The presented setup is integrated in a Tescan Lyra FEG high resolution microscope with an additional focused ion beam unit (FIB). On the one hand, the FIB is used to structure the gripper-jaws, on the other hand, it provides an imaging system with an additional point of view to the SEM's perspective which allows the collection of much more information about the system's pose.

\section{A. Robotic System}

The experimental setup mounted inside the SEM is developed with the objective of being versatile for different nanomanipulation and -handling tasks. Thus, the setup is modularly designed and consists of a coarse- and a finepositioning unit [10]. Each unit possesses three linear degrees of freedom while the coarsepositioning unit offers a maximal stroke of $35 \mathrm{~mm}$ in $\mathrm{x} / \mathrm{y}$-direction and $27 \mathrm{~mm}$ in $\mathrm{z}$-direction, the finepositioning unit offers a maximal stroke of $50 \mu \mathrm{m}$ in all directions. The actuators are equipped with internal optical sensors enabling a closed-loop accuracy of at least $500 \mathrm{~nm}$ and internal optical sensors enabling a closed-loop accuracy of at least $1.6 \mathrm{~nm}$, respectively.

According to the automation of the preassembly, the com(b) Combined pattern strategy involves circular milling with important areas being milled last (light blue), big areas are milled first (red). bination of coarse- and fine positioning units provides crucial benefits. High strokes allow mounting several reachable samples in the same setup, which is important for a fully automated preassembly process without any manual modifi-

Fig. 4: FIB etching patterns applying different scan strategies. cation of the setup. On the other hand, high accuracy allows 


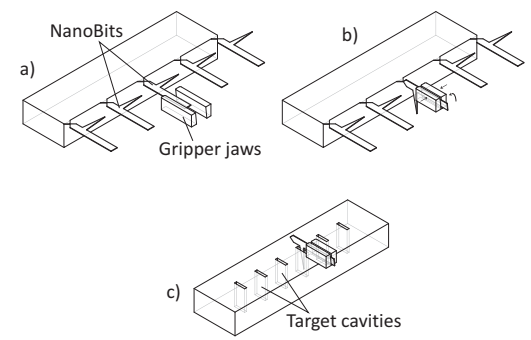

Fig. 6: Scheme of the NanoBit's detaching: a) The NanoBit is parallel to the substrate's surface. b) The NanoBit performs an out-of-plane turn and detaches from the substrate. c) The NanoBit is places in a cavity of the cartridge.

performing well-controlled movements in order to guarantee damage-free handling of the NanoBits.

\section{B. Handling Strategies}

During its application a NanoBit has to be transfered at least three times:

i) During the preassembly - from the fabrication substrate to the cartridge.

ii) During the application in the AFM - from the cartridge to the tip of the cantilever.

iii) At the disposal - cantilever has to be freed of the used NanoBit facilitating mounting of a new NanoBit there.

As mentioned above, simple pick-and-place transfer is not feasible in the nanometer scale and each transfer step reveals additional particular problems. Hence, all transfer techniques and tools have to be designed according to the actual task but still adjusted dependent on all other requirements given by the different steps.

1) NanoBit Gripping: The first handling step after the fabrication is detaching the NanoBit from the fabrication substrate. It can be performed straightforward by the gripper, since the NanoBit's suspension serves as predetermined breaking point, which allows detaching by the application of very small forces.

As the second step, the tip of the NanoBit is inserted into a cavity of the cartridge, which requires that the NanoBit's tip be perpendicular to the substrate's surface. The consequential $90^{\circ}$ turn of the NanoBit can not be achieved by turning the entire gripper, since the gripper's geometry would not allow the approach to the fabrication substrate. Thus, an out-of-plane turn inside the gripper jaws is desired, which can be achieved by special structured gripper jaws for example. This strategy facilitates a fast and seamless gripping process (see Figure 6).

Generally, if standard grippers with parallel gripping-planes clamp a NanoBit, it will turn in the jaws at some time of the handling process, either clockwise or counter-clockwise. In order to benefit from this spontaneous effect, tilted gripper jaws can be used to give the NanoBit the preferred turning direction. Two different approaches are used which are based on a configuration, in which the NanoBit's plane and the gripper's jaw plane are already slightly tilted (see Figure 7):

i) The gripper is parallel to the substrate's surface and only the gripping surfaces are tilted by about $5^{\circ}$. This
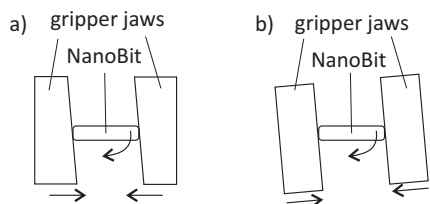

Fig. 7: Different gripping strategies: a) The inner face of the gripping jaws is tilted only. b) The entire gripper unit is tilted, which effects the closing direction, too.

configuration can not be directly achieved in the manufacturing process of the gripper which makes an additional structuring of the gripper necessary. A rotational momentum at the NanoBit occurs caused by the nonsymmetric clamping. The advantage is a non-tilted configuration of the entire gripper and substrate preserving an environment without any constraints.

ii) The entire gripper unit is mounted tilted, while the angle is limited by the geometry constraints. Compared to the first approach, the advantage in this setup is, that the actuation direction of the gripper jaws is tilted as well. Thus, the NanoBits turning becomes even more probable, since the actuation treats the NanoBit with an actual rotational momentum.

For both approaches, the rotational momentum caused by closing the gripper jaws is more than sufficient to turn the NanoBit completely and detach it from the fabrication substrate at the predetermined breaking point.

Regardless of the approach, a FIB treatment of the gripper jaws is beneficial to the assembly process and especially the automation. Even if the gripping planes are not tilted, tapering the gripper's tips is very useful. It allows a much better estimation of the grippers position in the on-top-view during the entire preassembly process and consequently facilitates better z-positioning and reliable automation. Figures 8 and 9 show SEM images of a FIB-structured thermoelectrical gripper. The inside of the gripper jaws are tilted and the tips are tapered.

Figure 9 shows the handling sequence of the preassembly process. Firstly, gripper and NanoBit have to be aligned, a further closing of the gripper-jaws causes the NanoBit to turn

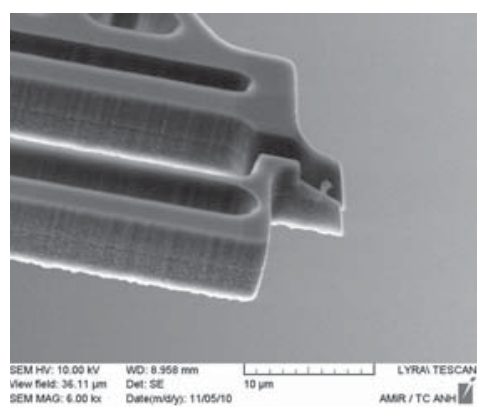

Fig. 8: SEM image of a FIB structured gripper. The gripping planes are tilted by $5^{\circ}$ and the tips are tapered. 


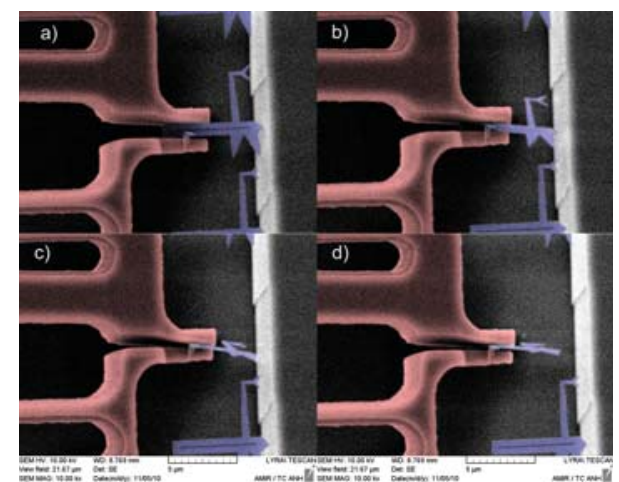

Fig. 9: SEM images of the NanoBit preassembly sequence: a) Both jaws are touching the NanoBit. b) Closing of the jaws causes the NanoBit to turn (in this case tip turns upwards). c) The NanoBit turns completely and detaches from the substrate. d) The gripper can remove the detached NanoBit.

to the point of a full $90^{\circ}$ detaching the NanoBit from the substrate. The closed gripper holds the NanoBit preventing an unintended bouncing during the break off. This strategy is performed with FIB-structured grippers as well as tiltmounted grippers. Both approaches work quite well, while the tilted setup reveals more reliable results concerning the determination of the turning direction.

2) Preassembly: Placing the NanoBit in the cartridge is the most crucial step of the preassembly sequence, since it has to be performed allowing for parasitic forces in the nanometer scale - most of all adhesion and van der Waals linkage. Due to the tiny mass of the NanoBits, the gripper can open its jaws without obtaining a disengagement by gravity of gripper and NanoBit. For this reason, the cartridge already has to be designed in a way, that it can assist the gripper with placing and disengaging the NanoBit by overbearing the parasitic forces. On the other hand, it is of vital importance that the tip of the NanoBit remains undamaged until it is used. For this reason, the NanoBit's tip is not allowed to touch any part of the cartridge during the preassembly process as well as the storage or transportation time. Therefore, the most adequate type of cavity is a narrow trench in a thin membrane. The dimensions of these trenches are about $200-300 \mathrm{~nm}$ in width and few $\mu \mathrm{m}$ in length, which guarantees that the tip of the NanoBit is already outside of the membrane and completely free ensuring contactless storage condition.

Figure 10 shows the placing strategy of NanoBit and cartridge. Firstly, the NanoBit has to be aligned above the cavity. The NanoBit is lowered subsequently until the NanoBit's body and handle rest on the cartridge. Secondly, the gripper is opened and withdrawn. Since the NanoBit rests in the cavity, it will be detracted from the gripper jaws and stay in the cartridge.

The experimental realization of this sequence is shown in Figure 11. After the clamped NanoBit is inserted into the cavity (Fig. 11a), the opened gripper can be retracted parallel to the cartridge's surface (Fig. 11b). The left NanoBit rests in

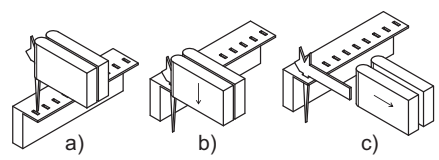

Fig. 10: Strategy for placing of NanoBit into the cartridge. a) The NanoBit is aligned above the cavity. b) The gripper is lowered inserting the NanoBit into the cavity. c) The NanoBit is stuck in the cavity, while the opened gripper is withdrawn.

the cavity, it is freestanding and accessible in the cavity (Fig. 11c).

3) Tip Exchange in the AFM: In order to address a wide application field, the in-situ exchange of NanoBits is highly aspired. This does not only concern the assembly of NanoBit and cantilever but also the release of the used NanoBit clearing the cantilever. The in-situ exchange demands several requirements:

i) All assembly steps must not involve complex joiningtechnologies.

ii) All assembly steps have to be reliable, damage free and verifiable. Especially, the NanoBit's release has to clear the Cantilever without any remainders.

iii) The AFM in-situ exchange declares the SEM usage impossible. All assembly steps have to be feasible with light-optical or without optical feedback.

In any event, the assembly of NanoBit and cartridge is realized without any adhesive or additional force. Hence, the transfer to the cantilever needs a little bonding force only and can be realized by mechanical pinching. The entire sequence of the in-situ exchange is shown in Figure 12. The cantilever features a small conical trench at its end, which is pressed against the NanoBit from the top (Fig. 12a). During all the application steps, the NanoBit remains in the trench due to the mechanical pressure. After using, the cantilever, it can be freed from the NanoBit utilizing additional cavities with deep conical trenches. The cantilever can strip itself of the NanoBit in these trenches, because they offer a much higher contact surface to the NanoBit than the cantilever's trench (Fig. 12c). Thus, reliable stripping can be realized.

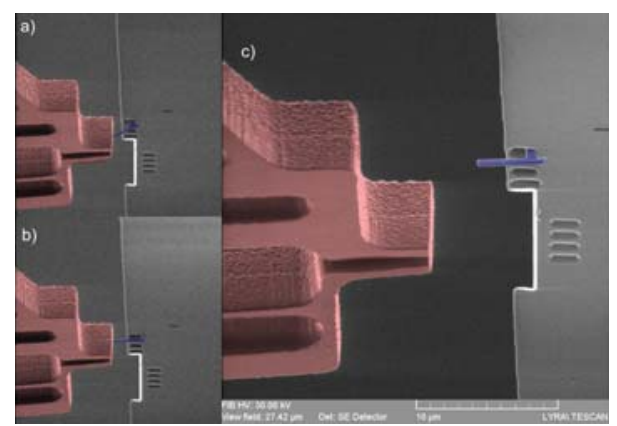

Fig. 11: FIB image of the placing sequence. a) The NanoBit is placed exactly in the cavity. b) The gripper is withdrawn parallel to the cartridge. c) The NanoBit rests in the cavity. 


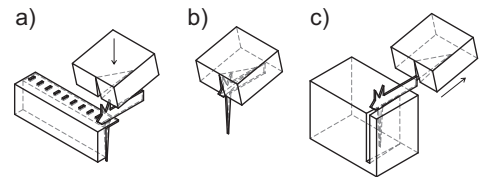

Fig. 12: AFM-side usage sequence: a) The cantilever's trench is pressed on the NanoBit. b) The NanoBit remains in the trench due to the mechanical pressure. c) The NanoBit is removed by an additional trench.

\section{Automation of NanoBits Assembly}

To make the NanoBit's preassembly viable for industrial application, as described above, the process needs to be fully automated. Furthermore, the automation aims for a throughput of at least 6 components per minute. There are two main process steps involved in this automation: a) Detaching a NanoBit from the production substrate and b) putting a NanoBit into a target cavity. Keeping these steps in mind, the crucial challenges are the alignment of the NanoBit and gripper, and the NanoBit and cavity, respectively. These challenges need to be solved through the automation.

\section{A. X/Y-Alignment}

Firstly, objects need to be aligned in the $\mathrm{x}$ - and y-plane. In the gripping sequence, accuracy higher than $100 \mathrm{~nm}$ is necessary, for the cartridge placing sequence even $50 \mathrm{~nm}$ are hardly sufficient in order to guarantee a damage-free assembly. In the handling setup, the precise location of the employed gripper, the NanoBits and the target cavities can only be determined using the SEM's visual feedback. The robots' internal sensors cannot guarantee movement with sufficient precision, due to temperature drift caused by the thermal gripper as well as electrostatic charging of the NanoBits. Thus, visual servoing is used for closed-loop positioning. In order to fulfill the throughput requirements, line scan-based tracking [11] is used to facilitate high-speed positioning. With this approach, the gripper can be aligned precisely with respect to a NanoBit in a few tens of milliseconds.

\section{B. Z-Alignment}

Secondly, a precise $\mathrm{z}$ alignment needs to be performed, which is much more complex due to the total lack of depth information from the SEM. Thus, two different approaches are used, dependent on the process step.

1) Alignment of gripper and NanoBit: To detach a NanoBit, the gripper is first aligned in the $\mathrm{x} / \mathrm{y}$-plane so that it is above the NanoBit and the NanoBit is visible between the gripper jaws. Then, the gripper is lowered until significant shadowing appears on the NanoBit. This shadow-based depth detection capitalizes on the fact that most secondary electrons hit the gripper and do not reach the electron detector when the NanoBit is located exactly between the gripper jaws [12].

2) Alignment of NanoBit and cartridge: To release the NanoBit into a cavity, the tip of the NanoBit within the closed gripper is positioned exactly over the cavity. Then, when the gripper is lowered, the handle of the NanoBit will come into contact with the edge of the cavity leading to slight bending. This bending can be rapidly detected by the line scan-based tracking and the NanoBit can be released.

\section{CONCLUSION}

The robotic preassembly is a reliable approach to achieve the realization of an AFM system using exchangeable scanning tips. Several crucial steps are identified and corresponding handling sequences proposed. The handling of NanoBits using structured or tilted grippers, and the placement of NanoBits in cavities are revealed to be promising techniques for the realization of NanoBit cartridges. The experiments have shown, that further automation is definitely feasible, due to well known experimental conditions and parameters of all objects.

\section{ACKNOWLEDGMents}

This work is supported by the European Commission in frame of the project NanoBits (257244).

\section{REFERENCES}

[1] J. Brugger, R. A. Buser, and N. F. de Rooij, "Silicon cantilevers and tips for scanning force microscopy," Sensors and Actuators A, vol. 34, no. 3, pp. 193-200, 1992

[2] P. A. Rasmussen, J. Thaysen, S. Bouwstra, and A. Boisen, "Modular design of AFM probe with sputtered silicon tip," Sensors and Actuators A: Physical, vol. 92, no. 1-3, pp. 96 - 101, 2001. [Online]. Available: http://www.sciencedirect.com/science/article/B6THG43HJVDR-G/2/5c1627a4ed2fa1110418a0b61423d6dc

[3] Y. Wang and D. W. van der Weide, "Microfabrication and application of high-aspect-ratio silicon tips," Journal of Vacuum Science \& Technology B: Microelectronics and Nanometer Structures, vol. 23, pp. 1582 - 1584, 2009.

[4] V. Eichhorn, S. Fatikow, D. Jasper, and P. Bøggild, "NanoBits: Exchangeable and customizable scanning probe tips as a versatile tool for nanoscale applications," in Proc. of International Workshop on Microfactories, Daejeon, Korea, October 2010.

[5] V. Eichhorn, S. Fatikow, T. Wortmann, C. Stolle, C. Edeler, D. Jasper, O. Sardan, P. Bøggild, G. Boetsch, C. Canales, and R. Clavel, "NanoLab: A Nanorobotic System for Automated Pick-and-Place Handling and Characterization of CNTs," in Proceedings of the IEEE International Conference on Robotics and Automation (ICRA 2010), Kobe, Japan, 2009, pp. 1826-1831

[6] R. T. Rajendra Kumar, S. U. Hassan, O. Sardan Sukas, V. Eichhorn, F. Krohs, S. Fatikow, and P. Bøggild, "Nanobits: customizable scanning probe tips," Nanotechnology, vol. 20, no. 39, p. 395703 (6pp), 2009.

[7] A. Lei, D. H. Petersen, T. J. Booth, L. V. Homann, C. Kallesoe, O. S. Sukas, Y. Gyrsting, K. Molhave, and P. Boggild, "Customizable in situ TEM devices fabricated in freestanding membranes by focused ion beam milling," Nanotechnology, vol. 21, no. 40, p. 405304, 2010 [Online]. Available: http://stacks.iop.org/0957-4484/21/i=40/a=405304

[8] B. Malm, D. H. Petersen, A. Lei, T. J. Booth, L. V. Homann, and P. Bøggild, "Optimization of FIB milling for rapid NEMS prototyping," Microelectronic Engineering, 2010, in press.

[9] S. Fatikow, Automated Nanohandling by Microrobots, ser. Springer Series in Advanced Manufacturing. Springer, 2008.

[10] V. Eichhorn, M. Bartenwerfer, and S. Fatikow, "Nanorobotic Strategy for Nondestructive Mechanical Characterization of Carbon Nanotubes," Bentham Journal Micro and Nanosystems, vol. 2, no. 1, pp. 32-37, 2010.

[11] D. Jasper, "High-speed Position Tracking for Nanohandling inside Scanning Electron Microscopes," in Proc. of IEEE Intl. Conference on Robotics and Automation (ICRA), Kobe, Japan, May 2009, pp. 508-513.

[12] V. Eichhorn, S. Fatikow, C. Dahmen, C. Edeler, C. Stolle, and D. Jasper, "Automated Microfactory inside a Scanning Electron Microscope," in Proc. Int. Workshop on Microfactories (IWMF'08), Evanston, USA, October 2008. 